\title{
Interleukin-6 induces an epithelial-mesenchymal transition phenotype in human adamantinomatous craniopharyngioma cells and promotes tumor cell migration
}

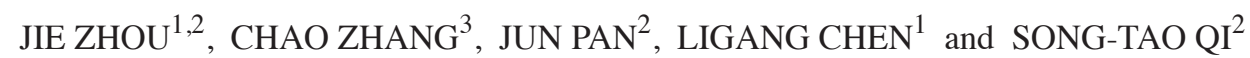 \\ ${ }^{1}$ Department of Neurosurgery, Affiliated Hospital of Southwest Medical University, Luzhou, Sichuan 646000; \\ ${ }^{2}$ Department of Neurosurgery, Nanfang Hospital, Southern Medical University, Guangzhou, Guangdong 510000; \\ ${ }^{3}$ Department of Pathology, Sun Yat-Sen University Cancer Center, Guangzhou, Guangdong 510060, P.R. China
}

Received February 28, 2016; Accepted March 1, 2017

DOI: $10.3892 / \mathrm{mmr} .2017 .6538$

\begin{abstract}
Total resection of adamantinomatous craniopharyngioma $(\mathrm{ACP})$ is complex and often leads to postoperative recurrence. This is due to the tendency of the tumor to invade the surrounding brain tissue and the generation of a local inflammatory state between the tumor cells and parenchyma. While there is evidence to suggest that interleukin-6 (IL-6) induces craniopharyngioma (CP)-associated inflammation, particularly in ACP, the role of IL- 6 in the progression of ACP remains unclear. The results of the present study demonstrated that CP inflammation was associated with pathological classification, extent of surgery, degree of calcification and postoperative hypothalamic status scale. Cytokine antibody arrays were conducted to measure the expression of IL-6 and other inflammatory factors in tumor tissues in response to various levels of inflammatory exposure. IL-6, IL-6 receptor (IL-6R) and glycoprotein 130 expression was detected by immunohistochemistry. In addition, an ELISA was performed to quantify the levels of soluble IL-6R (sIL-6R) in the cystic
\end{abstract}

Correspondence to: Dr Ligang Chen, Department of Neurosurgery, Affiliated Hospital of Southwest Medical University, 25 Taiping Street, Luzhou, Sichuan 646000, P.R. China

E-mail: chengligang.cool@163.com

Dr Song-Tao Qi, Department of Neurosurgery, Nanfang Hospital, Southern Medical University, 1838 Guangzhou Road, Guangzhou, Guangdong 510000, P.R. China

E-mail: zj000718@yeah.net

Abbreviations: ACP, adamantinomatous craniopharyngioma; CPs, craniopharyngiomas; EMT, epithelial-mesenchymal transition; HPF, high-power field; HSS, hypothalamic status scale; IL-6, interleukin-6; IL-6R, IL-6 receptor; sIL-6R, soluble IL-6R; SPCP, squamous papillary craniopharyngioma; TAFs, tumor-associated fibroblasts

Key words: craniopharyngioma, interleukin-6, inflammation, migration, epithelial-mesenchymal transition fluid and supernatants of ACP cells and tumor-associated fibroblasts. These measurements demonstrated that ACP cells produce IL- 6 and its associated proteins. In addition, the results revealed that while the viability of ACP cells was not affected, the migration of ACP cells was promoted by IL-6 treatment in a concentration-dependent manner. Conversely, treatment with an IL-6-blocking monoclonal antibody significantly decreased the migration of ACP cells. In addition, IL-6 treatment increased the expression of vimentin and decreased the expression of E-cadherin in a dose-dependent manner. The findings of the present study demonstrate that IL-6 may promote migration in vitro via the classic- and trans-signaling pathways by inducing epithelial-mesenchymal transition in ACP cell cultures.

\section{Introduction}

Craniopharyngiomas (CPs) are benign epithelial tumors of the sellar region that arise along the path of the craniopharyngeal duct. CPs are histopathologically classified into two subtypes: Adamantinomatous craniopharyngioma (ACP) and squamous papillary craniopharyngioma (SPCP). ACP often forms finger-like protrusions that invade the surrounding brain tissue, inducing a local inflammatory state between the tumor cells and parenchyma. Therefore, total resection of the tumor is complex and can lead to postoperative recurrence and a poorer clinical prognosis when compared to SPCP (1).

Inflammation is a critical component of tumor progression. It is now evident that the tumor microenvironment, which is largely composed of inflammatory cells, is an indispensable participant in the neoplastic process, stimulating proliferation, survival and migration (2). In some cases of ACP, infiltration of neutrophils, lymphocytes and eosinophils has been detected in the tumor mass or the surrounding parenchyma. In addition, spontaneous rupture of ACP cysts and spillage of cyst fluid during surgery can lead to aseptic meningitis (3). Inflammatory cells and the cytokines secreted may modulate the local immune response and affect tumor cell growth. High levels of IL- 6 have been detected in cyst fluid and CP tissues; a previous study indicated that IL-6 served an important role in the inflammatory reaction that occurred in the interface 
between the $\mathrm{CP}$ and the brain parenchyma (4). In addition, in some cancer cells, IL-6 acts as an autocrine or paracrine factor that promotes tumor cell proliferation, migration and invasion in vitro (5). These findings indicate that IL-6 may exert a biological influence on ACP cells.

It has previously been demonstrated that IL- 6 induces an epithelial-mesenchymal transition (EMT) phenotype in breast cancer cells, promoting migration and invasion (6). Our previous research revealed that EMT may serve a role in the pathogenesis and development of $\mathrm{CP}$, particularly ACP (7). However, the role of IL-6, particularly in association with EMT, in ACP progression is unclear. The present study investigated the biological role and significance of IL- 6 in ACP cells using cell culture assays of primary human ACP cells. Activation of the classic and trans-signaling pathways of IL-6 was revealed to induce an EMT phenotype to promote the migration of human ACP cells; however, IL-6 did not affect the viability of ACP cells.

\section{Materials and methods}

Patients and tissue specimens. A prospective cohort of study patients were selected for clinicopathological analysis according to the inclusion and exclusion criteria as described previously (7). A total of $49 \mathrm{CP}$ biopsy specimens (37 ACPs and 12 SPCPs) were obtained from the archives of the Department of Neurosurgery at the Nanfang Hospital, Southern Medical University (Guangzhou, China) for use in the present study. The biopsy specimens were originally collected between January 2005 and January 2009. Patient age ranged between 4 and 60 years, with a mean of 29.39 \pm 18.04 years. Cystic fluid samples were obtained at surgery from 6 of the patients with cystic $\mathrm{ACP}$, and stored at $-80^{\circ} \mathrm{C}$.

A total of 13 solid ACP specimens were obtained at surgery from the Department of Neurosurgery at the Nanfang Hospital, Southern Medical University for primary ACP and tumor associated fibroblast (TAF) cell culture, between July 2010 and March 2012. Patient age ranged between 3 and 50 years, with a mean of $21.77 \pm 16.60$ years. The present study was approved by the Affiliated Hospital of Southwest Medical University (Luzhou, China). Patients provided written informed consent.

Inflammatory density score. Specimens removed at surgery were immediately fixed in $10 \%$ formalin, and subsequently embedded in paraffin. Sections were subsequently stained with hematoxylin and eosin (both from ZSGB-Bio, Beijing, China) for diagnosis and assessment of the inflammatory density score. The inflammatory density score was determined by counting the number of inflammatory cells adjacent to the interface between the $\mathrm{CP}$ and the surrounding normal tissue, to a depth corresponding with 1 high-power field (HPF magnification, x400) in 10 consecutive fields. Inflammatory density was graded on a 4-grade scale: Grade 0, no inflammation; grade $1,<15$ cells/field; grade 2, 15-50 cells/field and grade 3, $>50$ cells/field. Density was recorded separately for each HPF and the inflammatory score for each case was calculated as the average of all HPFs examined. Cases were divided into three groups according to the inflammatory score: Grades 0-1, mild; grade 2, moderate and grade 3, severe. Slides were analysed with an Olympus BX-51 microscope with a DP-71 CCD camera (both from Olympus Corporation, Tokyo, Japan).

Evaluation of calcification. The degree of CP calcification in biopsy specimens from the 49 patients was determined using the criteria described in our previous study (8). Briefly, tumor calcification was identified as areas of high attenuation on computerized tomography scans or as low signal (usually) on magnetic resonance images. Tumors were classified as follows: Calcification absent (-); solid lumps/a little calcification or eggshell pattern lining the cyst wall $(+$, mild to moderate calcification); popcorn-like foci (++, severe calcification).

Follow-up. The postoperative hypothalamic status scale (HSS) of biopsy specimens from the 49 patients was determined 6 months after surgery using the criteria described by Fahlbusch et al (9): I, good (without any new permanent neurological, neuropsychological or endocrinal deficits); II, moderate (with new endocrine deficits requiring permanent replacement therapy); III, fair (with neurological or neuropsychological deficits, with autonomy); IV, poor (severe neurological and/or hypothalamic disturbances with total dependency, or mortality).

Cytokine antibody array. An antibody-based cytokine array system was used to detect the levels of inflammatory cytokines in frozen ACP tissues obtained at surgery belonging to the mild and severe inflammatory groups, which had been stored in liquid nitrogen. The experiment was conducted using the Human Inflammation Antibody Array III kit (AAH-INF-G3; RayBiotech, Inc., Norcross, GA, USA) according to the manufacturer's protocol.

ACP cell culture. Primary ACP cell cultures were established using solid tumor specimens obtained at surgery from the Department of Neurosurgery at the Nanfang Hospital, Southern Medical University (Guangzhou, China) in a similar manner as described by Hölsken et al (10). Briefly, a portion $\left(1-10 \mathrm{~cm}^{3}\right)$ of the tumor sample was placed in Dulbecco's modified Eagle's medium (DMEM; Gibco; Thermo Fisher Scientific, Inc., Waltham, MA, USA) containing penicillin/streptomycin (100 units/ml/100 $\mu \mathrm{g} / \mathrm{ml}$, Sigma-Aldrich; Merck KGaA, Darmstadt, Germany) and 10\% fetal bovine serum (FBS; Gibco; Thermo Fisher Scientific, Inc.) immediately following surgical removal. Tumor tissue was subsequently separated into small pieces, washed in phosphate-buffered saline (PBS) containing penicillin, streptomycin (100 units/ml/100 $\mu \mathrm{g} / \mathrm{ml})$, trypsin and EDTA (0.25 and 0.05\%; Gibco; Thermo Fisher Scientific, Inc.) and incubated for $20-25 \mathrm{~min}$ at $37^{\circ} \mathrm{C}$ and $5 \% \mathrm{CO}_{2}$. A culture solution was prepared using DMEM and DMEM/F12 (1:1; Gibco; Thermo Fisher Scientific, Inc.) in a final ratio of $3: 1$, to which insulin (5 $\mu \mathrm{g} / \mathrm{ml}$; Sigma-Aldrich; Merck KGaA), transferrin (5 $\mu \mathrm{g} / \mathrm{ml}$; EMD Millipore, Billerica, MA, USA), T3 (2x10-9 mol/l; Sigma-Aldrich; Merck KGaA), hydrocortisone $(0.4 \mu \mathrm{g} / \mathrm{ml}$; Sigma-Aldrich; Merck KGaA), cholera toxin (2x10-10 mol/1; Sigma-Aldrich; Merck KGaA), antibiotics (penicillin/streptomycin, 100 units $/ \mathrm{ml} / 100 \mu \mathrm{g} / \mathrm{ml}$ ), fungizone ( $2.5 \mu \mathrm{g} / \mathrm{ml}$; Gibco; Thermo Fisher Scientific, Inc.), L-glutamine (2 mM; Gibco; Thermo Fisher Scientific, Inc.) and 10\% FBS were added. Following 2 days of culture, epidermal growth 
factor (EGF; $10 \mu \mathrm{g} / \mathrm{ml}$; R\&D Systems, Inc., Minneapolis, MN, USA) was added to the culture medium and the medium was renewed every second day and supplemented with EGF. Based on observations of cell morphology and immunohistochemical staining for pan-keratin (CK; 1:500, \#4545, Cell Signaling Technology, Inc., Danvers, MA, USA), the cultures that contained the most homogenous epithelial-like cells were expanded. Cell morphology was analysed with a Leica DMIRE2 inverted microscope (Leica Microsystems GmbH, Wetzlar, Germany).

TAF culture and analysis. The ACP tumor tissues obtained at surgery from Nanfang Hospital were carefully dissected, minced with scalpels, then transferred to new culture dishes with a little DMEM. Following $4 \mathrm{~h}\left(37^{\circ} \mathrm{C}, 5 \% \mathrm{CO}_{2}\right)$, the tumor tissues were cultured in DMEM supplemented with penicillin/streptomycin (100 units $/ \mathrm{ml} / 100 \mu \mathrm{g} / \mathrm{ml})$ and 10\% FBS. Following 1 week of culture, the cells that had migrated from the tissue clumps were trypsinized, transferred to new culture dishes and left for a short period (10-20 min) to allow for surface attachment. Cells that had not attached were then removed and the dishes with firmly attached cells were washed with PBS to further eliminate loosely attached cells. Based on observations of cell morphology and positive staining for the fibroblast markers vimentin (1:500; 550513; BD Biosciences, Inc., Franklin Lakes, NJ, USA) and $\alpha$-SMA (1:100; bs-10196R; BIOSS, Beijing, China) and negative staining for the epithelial cell marker Pan-CK (dilution, 1:500, \#4545, Cell Signaling Technology, Inc.), the cultures that contained the most homogenous fibroblastic-like cells were expanded. The culture supernatant from the 3rd passage was then harvested for ELISA. Cell morphology was analysed with a Leica DMIRE2 inverted microscope.

Protein preparation and immunoblotting. Total proteins from tumor tissue stored in liquid nitrogen, obtained at surgery from the Department of Neurosurgery at the Nanfang Hospital, Southern Medical University, were isolated in lysis buffer (Beyotime Institute of Biotechnology, Shanghai, China). The concentration of each fraction was evaluated by photometric measurement (wavelength, $562 \mathrm{~nm}$; Tecan Group Ltd., Männedorf, Switzerland) using a bicinchoninic acid assay kit (Nanjing KeyGen Biotech Co., Ltd., Nanjing, China). Following 10\% SDS-PAGE (50 $\mu \mathrm{g} /$ lane), separated proteins were transferred to polyvinylidene difluoride membranes. The membranes were blocked in 5\% skim milk in TBS buffer

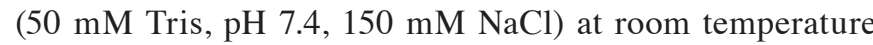
for $1 \mathrm{~h}$. The membranes were then probed with antibodies against IL-6 (1:400; ab9324; Abcam, Cambridge, UK), IL-6R (1:400; BA0992; Wuhan Boster Biological Technology, Ltd., Wuhan, China), vimentin (1:500; 550513; BD Biosciences, Inc.), E-cadherin (1:1,000; \#4065; Cell Signaling Technology, Inc.) and $\beta$-actin (1:10,000; SAB4200248; Sigma-Aldrich; Merck $\mathrm{KGaA}$ ) overnight at $4^{\circ} \mathrm{C}$, and thereafter with horseradish peroxidase (HRP)-conjugated rabbit anti-mouse/goat anti-rabbit secondary antibodies (1:4,000; Southern Biotech, Birmingham, AL, USA), at room temperature for $1 \mathrm{~h}$. The protein bands were detected by chemiluminescence using Immobilon Western Chemiluminescence HRP substrate (EMD Millipore).
Immunohistochemical staining. Immunohistochemical staining was performed using the 2 -step plus poly-HRP method as described previously (7). Briefly, specimens removed at surgery from the Department of Neurosurgery at Nanfang Hospital, Southern Medical University, were fixed immediately in $10 \%$ formalin, and subsequently embedded in paraffin wax. One representative section of tissue from each patient was cut to $4 \mu \mathrm{m}$ and placed on poly-L-lysine-coated slides. The slides were deparaffinized, rehydrated, immersed in $10 \mathrm{mM}$ sodium citrate buffer $(\mathrm{pH}$ 6.0) and pretreated in a microwave oven for $20 \mathrm{~min}$, followed by a $15 \mathrm{~min}$ rinse with PBS. Following blocking with $3 \%$ hydrogen peroxide for $10 \mathrm{~min}$ at room temperature, the slides were incubated at $4^{\circ} \mathrm{C}$ overnight with primary antibodies: Anti-IL-6 (1:200; ab9324; Abcam), anti-IL-6R (1:100; BA0992; Wuhan Boster Biological Technology, Ltd.) and anti-gylcoprotein 130 (GP130) (1:100; bs-1459R; Beijing Biosynthesis Biotechnology Co., Ltd., Beijing, China). The slides were then stained with the 2-step plus Poly-HRP Anti-Rabbit Immunoglobulin G (IgG) Detection system (PV-6001; ZSGB-Bio) for IL-6R and GP130, or 2-step plus Poly-HRP Anti-Mouse IgG Detection System (PV-6002; ZSGB-Bio) for IL-6. Following visualization of the reaction with 3,3'-diaminobenzidine, the slides were counterstained with hematoxylin at room temperature for $5 \mathrm{~min}$ and covered with a glycerin gel. Negative controls consisted of tissue sections incubated with PBS instead of the primary antibody. Slides were analysed with an Olympus BX-51 microscope equipped with a DP-71 CCD camera.

ELISA. ACP cells and TAFs were grown to subconfluence $\left(2.5 \times 10^{6}\right.$ cells $)$ and then incubated in serum-free DMEM for $24 \mathrm{~h}\left(37^{\circ} \mathrm{C}, 5 \% \mathrm{CO}_{2}\right)$. The supernatants were then harvested for ELISA. Cystic fluid samples were obtained at surgery from the Department of Neurosurgery at Nanfang Hospital, from 6 patients affected by cystic ACP, and stored in $-80^{\circ} \mathrm{C}$. To detect sIL-6R in the supernatant of ACP cells, TAFs or the cystic fluid, an ELISA kit (KA0523; Abnova, Taipei, Taiwan) was conducted according to the manufacturer's protocol.

\section{Migration assays}

Boyden chamber assay. A Boyden chamber migration assay was used to quantify the number of migratory cells (Costar; Corning Incorporated, NY, USA). ACP cells that had been serum starved for $6 \mathrm{~h}$ were resuspended in serum-free culture $\left(1 \times 10^{5}\right.$ cells $\left./ 300 \mu \mathrm{l}\right)$ medium containing IL-6 $(0-100 \mathrm{ng} / \mathrm{ml})$ or IL-6 (100 ng/ml)/anti-hIL-6- antibody (10 $\mu \mathrm{g} / \mathrm{ml}$; MAB206; R\&D Systems, Inc.) and placed in the top chamber of the inserts $\left(1 \times 10^{5}\right.$ cells/well). Following incubation for $20 \mathrm{~h}\left(37^{\circ} \mathrm{C}\right.$, $5 \% \mathrm{CO}_{2}$ ), the inserts were removed; cells were fixed and stained with Giemsa solution (Solarbio Life Sciences, Beijing, China) at room temperature for $20 \mathrm{~min}$, and were viewed and counted under a light microscope. Images of representative microscopic fields were captured. Quantification of cell migration was expressed as the mean count of stained cells in 5 random fields of each filter. Each experiment was repeated three times $(n=3)$.

Wound-healing assay. ACP cells ( $1 \times 10^{5}$ cells/well) were grown to confluence in 24-well plates. A uniform wound was made in each plate using a $200-\mu 1$ pipette tip, and the plates were 
washed with PBS and incubated in serum-free culture medium containing IL-6 (0-100 ng/ml) or IL-6 (100 ng/ml)/anti-hIL-6 -antibody $(10 \mu \mathrm{g} / \mathrm{ml})$. The wound area was observed with a Leica DMIRE2 inverted microscope, and images were immediately captured $48 \mathrm{~h}$ following wound generation. Experiments were repeated three times $(n=3)$.

Cell proliferation assay. ACP cells were resuspended in serum-free culture $\left(1 \times 10^{3}\right.$ cells/ $100 \mu \mathrm{l} /$ well $)$ medium containing IL-6 (0-100 $\mathrm{ng} / \mathrm{ml})$ and were seeded in 96-well plates. The 3-(4,5-dimethylthiazol-2-yl)-2,5-diphenyl-tetrazolium bromide (MTT; $20 \mu \mathrm{l} /$ well) assay was used to determine cell viability at $0,12,24$ and $48 \mathrm{~h}$ after seeding. The absorbance at $570 \mathrm{~nm}$ was measured using a Quant Universal Microplate Spectrophotometer (BioTek Instruments, Inc., Winooski, VT, USA).

Statistical analysis. All numerical data are presented as the mean \pm standard deviation, all experiments were repeated three times. Statistical differences between clinical variables and inflammatory scores were evaluated using the $\chi^{2}$ test or Fisher's exact test for categorical variables and the non-parametric Spearman correlation test for continuous variables. One-way analysis of variance was used to assess the significance of differences among experimental groups and controls. Multiple comparisons was performed using Fisher's least significant difference test. The results were analyzed using SPSS statistical software for Windows, version 13.0 (SPSS, Inc., Chicago, IL, USA). $\mathrm{P}<0.05$ was considered to indicate a statistically significant difference.

\section{Results}

Correlation of inflammatory density with clinicopathological characteristics. Inflammation was present in $77.6 \%$ of $\mathrm{CP}$ cases: Mild in 24 (49\%), moderate in 15 (30.6\%) and severe in $10(20.4 \%)$. A total of 11 cases in the mild group (11/49, $22.4 \%$ ) cases were free of inflammation. The associations between clinicopathological parameters and the inflammatory density score are presented in Table I. There were no statistically significant differences between the inflammatory density scores and age, sex, tumor size and recurrence. However, there was a statistically significant difference between the inflammatory density score and pathological classification $(\mathrm{P}=0.037)$, the extent of surgery $(\mathrm{P}=0.017)$, the degree of calcification $(\mathrm{r}=0.326, \mathrm{P}=0.022)$ and HSS $(\mathrm{r}=0.376, \mathrm{P}=0.008)$.

Protein expression levels of inflammatory cytokines in response to different levels of inflammation in ACP. Antibody arrays were used to investigate the expression profile of major inflammatory cytokines in ACPs in response to various levels of inflammatory exposure. Representative arrays analyzing tumor tissues in the mild and severe groups are shown in Fig. 1. The mean optical intensity of positive spots from tumor tissues was estimated. Among these, spots corresponding to the IL-6 and IL-6R molecules were the most strongly expressed in the severe group. The differential expression of IL-6 and IL-6R was further confirmed by western blot analysis using tissue lysates that were different from those employed in the antibody array analysis (Fig. 2A).
IL-6 and IL-6R are expressed in ACP tissues. Of tumor tissues from 10 cases of ACP, 70 (7/10) and 60\% (6/10) exhibited positive cytoplasmic immunoreactivity for IL-6 and IL-6R, respectively. Their immunostaining was predominantly identified within the connective tissue mesenchymal cells, the peripheral (basal palisaded) epithelial component and epithelial whorls of ACP tissues. Conversely, the central stellate reticulum components exhibited low or no immunoreactivity. Positive cytoplasmic immunoreactivity for GP130 was observed in $90 \%$ (9/10) of ACP tissues and was revealed to be equally distributed throughout the tumor. These results demonstrated that tumor and mesenchymal cells produce IL-6, indicating the presence of the IL-6 autocrine and/or paracrine systems in ACP (Fig. 2B-D).

\section{Establishment of primary ACP and TAF cultures}

$A C P$ culture. To investigate the influence of IL-6 on the biological characteristics of ACP cells, primary ACP cultures were established directly from six different CP biopsy tissues. All tumors were histologically classified as ACP, according to the 2007 World Health Organization classification system for tumors of the nervous system (11). From each tumor specimen, a primary ACP culture was prepared according to established procedures to selectively raise epithelial cells. The present study identified all of the ACP cells according to their immunoreactivity for Pan-CK, a well-documented ACP cell marker (10). Based on the epithelial-like morphology and the Pan-CK phenotype, the epithelial origin of ACP cell cultures was demonstrated (Fig. 3).

TAF culture. To determine the role of the tumor microenvironment in the biological characteristics of ACP cells, primary fibroblast cell cultures were established directly from three different ACP tumor biopsy tissues. The adherent cells, which possessed a fibroblast-like morphology, were successfully cultivated from ACP tissues. To confirm that these cells were fibroblasts and not contaminated with tumor cells, the adherent cells were assessed by immunohistochemistry using vimentin, $\alpha$-SMA and Pan-CK, which are specific for human fibroblasts. The adherent cells were positively stained for vimentin and $\alpha$-SMA, and negatively stained for Pan-CK (Fig. 4A-C).

sIL-6R expression in the supernatant of ACP cells, ACP fibroblasts and ACP cystic fluid. Since some tumor tissues exhibited no IL-6R expression, the expression of sIL-6R was also determined in the supernatants of ACP cells, ACP fibroblasts and ACP cystic fluid by ELISA. Various expression levels of sIL-6R were observed in the supernatants of ACP cells, ACP fibroblasts, and ACP cystic fluid (Fig. 4D-F). These results indicated that ACP cells and tumor associated fibroblast cells in the tumor microenvironment secrete the sIL-6R. The present study hypothesized that after binding to its receptors, IL- 6 may form a complex to activate the IL- 6 classic- and trans-signaling pathways, promoting alterations in tumor cell characteristics, thus affecting the prognosis of these patients.

IL-6 promotes the migration of ACP cell cultures. The effects of IL- 6 on the migration of ACP cell cultures was analyzed by Boyden chamber and wound-healing assays. As presented in Fig. 5, migration of ACP cells was enhanced by IL-6 treatment 
Table I. Evaluation of the associations between inflammatory density score and clinicopathological characteristics in craniopharyngioma.

Inflammatory density score

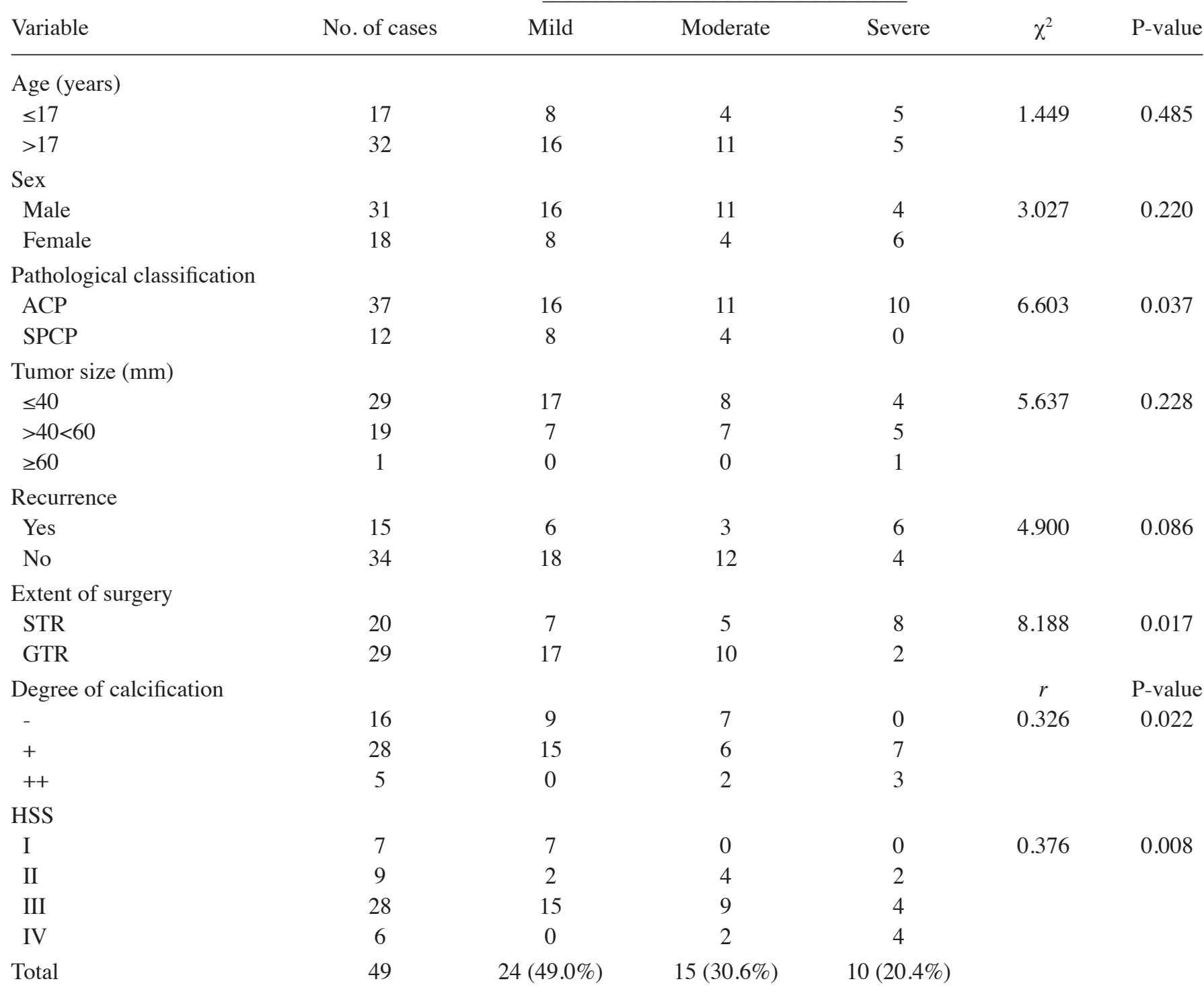

Inflammatory density scores: Mild, grades 0 (no inflammation) and 1 ( $<15$ inflammatory cells/field); moderate, grade 2 (15-50 inflammatory cells/field); severe, grade 3 (>50 inflammatory cells/field). ACP, adamantinomatous craniopharyngioma; SPCP, squamous papillary craniopharyngioma; STR, subtotal resection; GTR, gross total resection; HSS, hypothalamic status scale.

for $24 \mathrm{~h}$ in a dose-dependent manner. Since IL-6 was observed to promote the growth of some tumor cells (12), an MTT assay was performed to assess whether the viability-associated effects of IL-6 influenced the number of migratory cells of ACP. The results demonstrated that with an IL-6 dose range between 0 and $100 \mathrm{ng} / \mathrm{ml}$, there were no significant differences between the viability rates of cells treated for 12 or $24 \mathrm{~h}$ (data not shown). These results indicated that IL-6 may promote tumor cell migration. Furthermore, antibody-based blockade of IL- 6 decreased the migration of ACP cell cultures significantly (Fig. 5A and B).

IL-6 promotes an EMT phenotype of ACP cells. Our previous study demonstrated that EMT serves a potential role in the pathogenesis and development of CP, particularly ACP (7). The present study hypothesized that tumor cells undergoing EMT may exhibit increased motility contributing to the invasiveness of ACP, which usually leads to a worse prognosis than that for SPCP, in which this feature is absent. Some cytokines have been revealed to induce phenotypes consistent with EMT in transformed epithelial cells, as well as carcinoma cell lines, such as breast cancer (6). The present study analyzed whether the effects of IL-6 on the migration of ACP cell cultures were associated with markers of EMT. Following treatment with IL- 6 for 48 h, western blotting was performed to investigate the alterations in vimentin and E-cadherin expression in whole cell extracts. As presented in Fig. 6, the protein expression of vimentin was upregulated, 


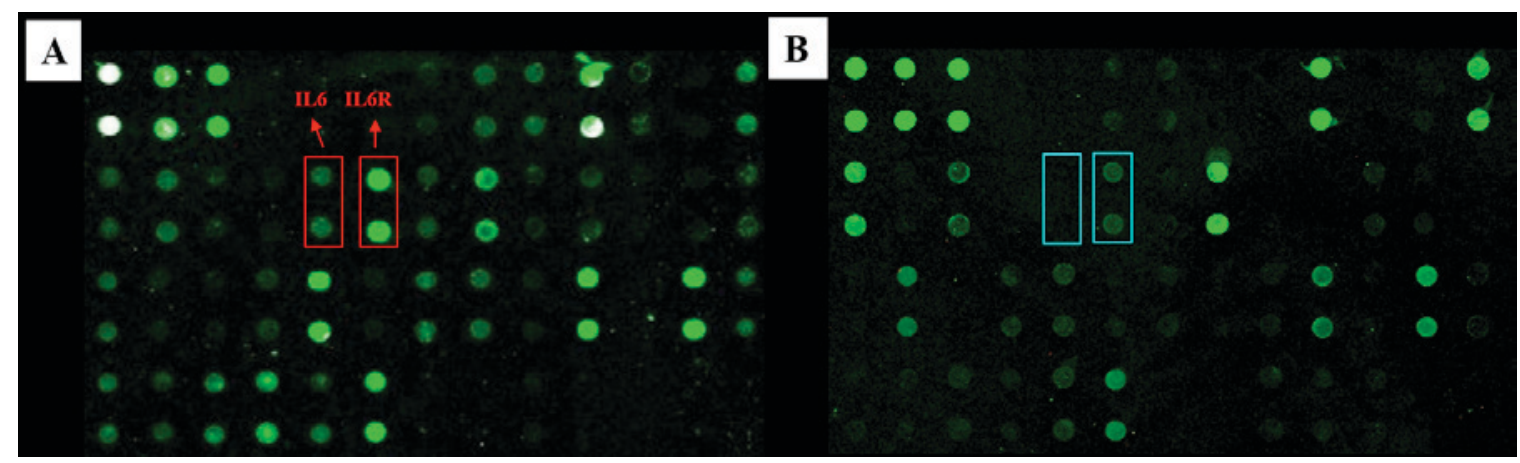

Figure 1. Antibody array of tissue lysates in response to various levels of inflammatory exposure in adamantinomatous craniopharyngioma. IL-6 and IL-6R were the most strongly expressed in (A) the severe group compared with (B) the mild group. IL-6R, interleukin-6 receptor; severe group, inflammatory density grade 3 ( $>50$ inflammatory cells/field); mild group, inflammatory density grades 0 (no inflammation) and 1 ( $<15$ inflammatory cells/field).

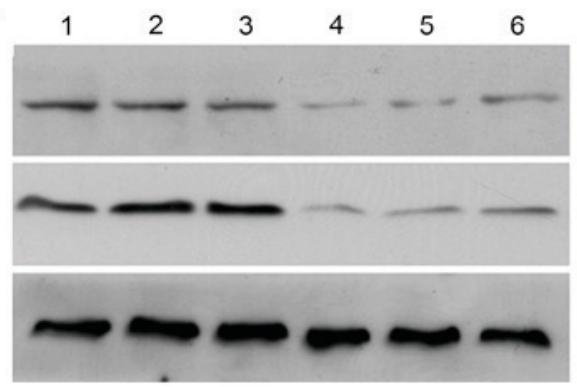

C

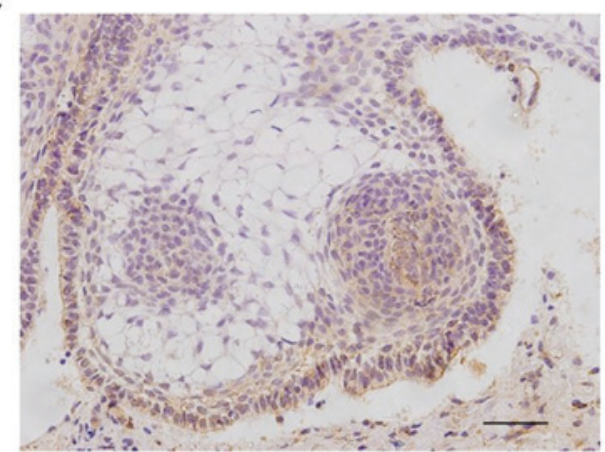

B

IL-6R
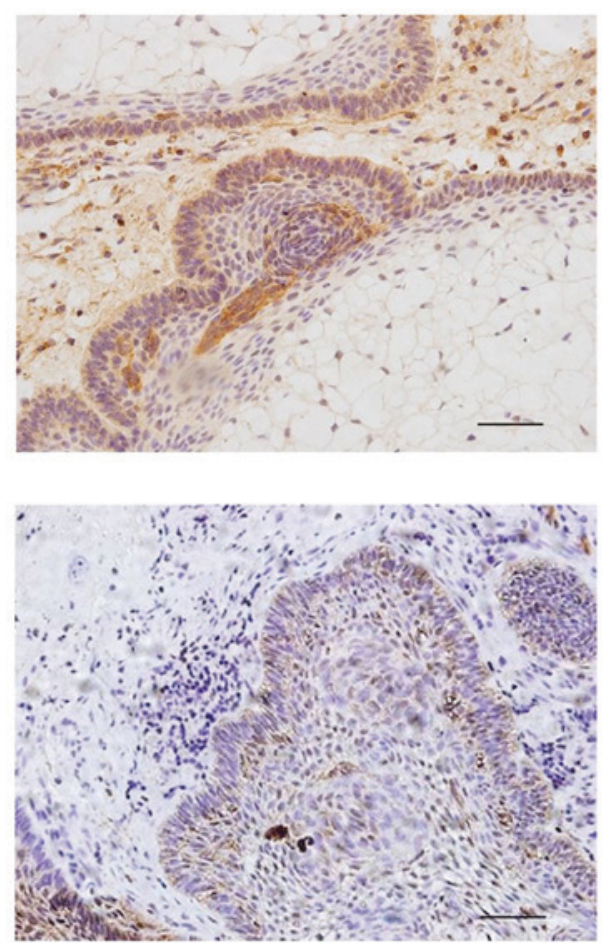

Figure 2. IL-6 was positively associated with tumor-induced inflammation. (A) Western blot analysis of IL-6 and IL-6R demonstrating the overexpression of IL-6 and IL-6R in the severe inflammation group when compared to the mild inflammation group, in accordance with the antibody array findings. Lanes 1-3: Severe inflammation group; lanes 4-6: Mild inflammation group. (B) IL-6, (C) IL-6R and (D) glycoprotein 130 expression in adamantinomatous craniopharyngioma tissues were examined using immunohistochemistry. Scale bars, $50 \mu \mathrm{m}$. IL-6R, interleukin- 6 receptor; severe group, inflammatory density grade 3 ( $>50$ inflammatory cells/field); mild group, inflammatory density grades 0 (no inflammation) and 1 ( $<15$ inflammatory cells/field).
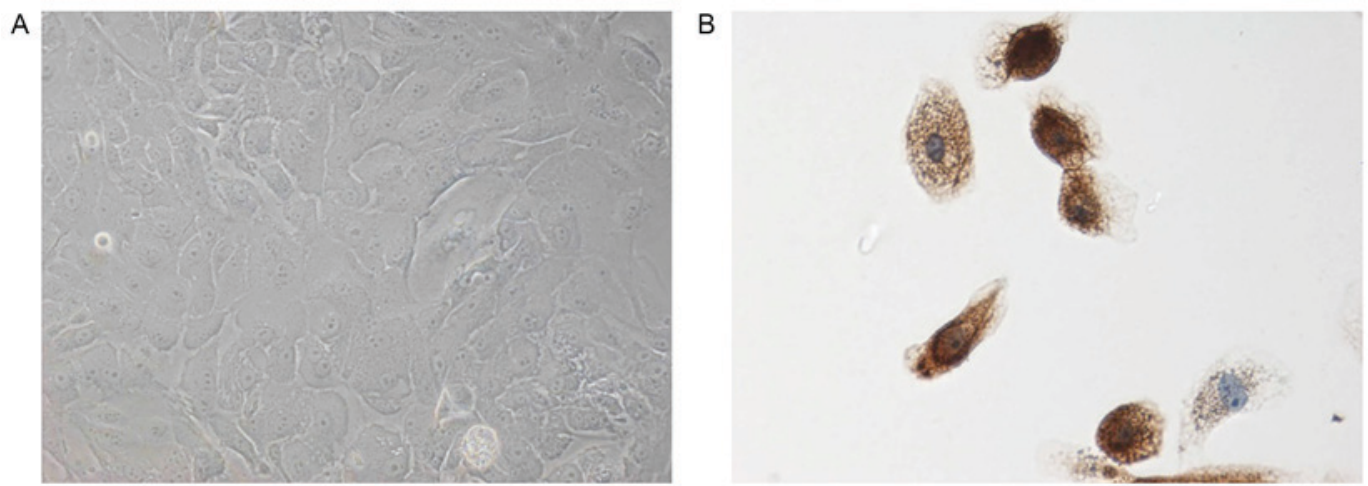

Figure 3. (A) Cobblestone-shaped primary cells of ACP in inverted phase (magnification, $\mathrm{x} 200$ ) and (B) Pan-CK immunostaining of primary ACP cells (magnification, x400). ACP, adamantinomatous craniopharyngioma. 

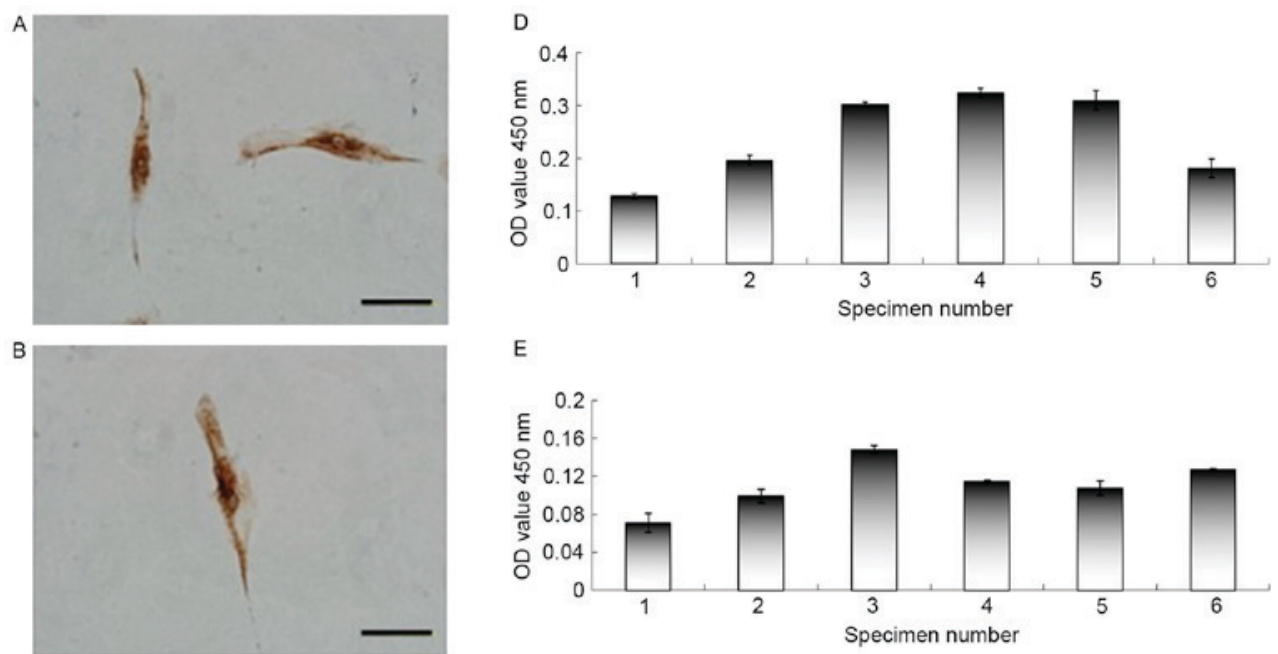

$\mathrm{E}$
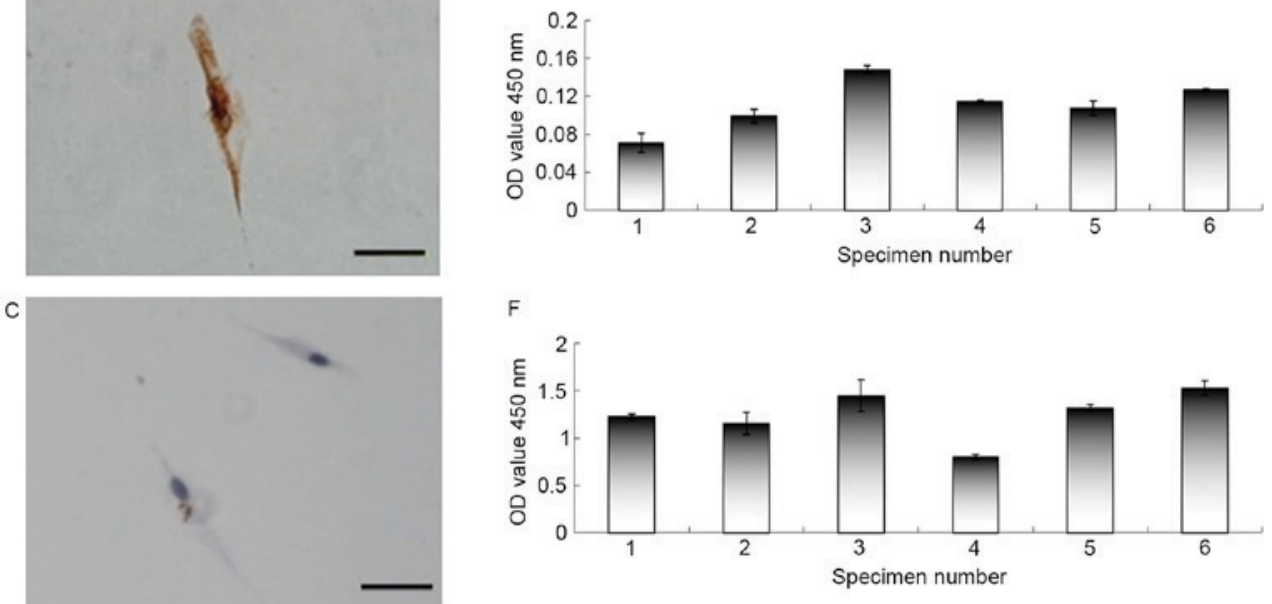

Figure 4. Cultured TAFs were identified and the levels of sIL-6R in the cystic fluid, and the supernatants of ACP cells and TAFs were quantified. The adherent TAF cells were positively stained for (A) vimentin and (B) $\alpha$-smooth muscle actin, and negatively stained for (C) Pan-CK (magnification, x400). Detection of sIL-6R in the supernatant of (D) ACP cells and (E) TAFs, and (F) the ACP cystic fluid from 6 randomly selected samples using ELISA. Values represent the mean \pm standard deviation, for 3 repeats. TAFs, tumor-associated fibroblast; sIL-6R, soluble IL-6 receptor; ACP, adamantinomatous craniopharyngioma; OD, optical density.

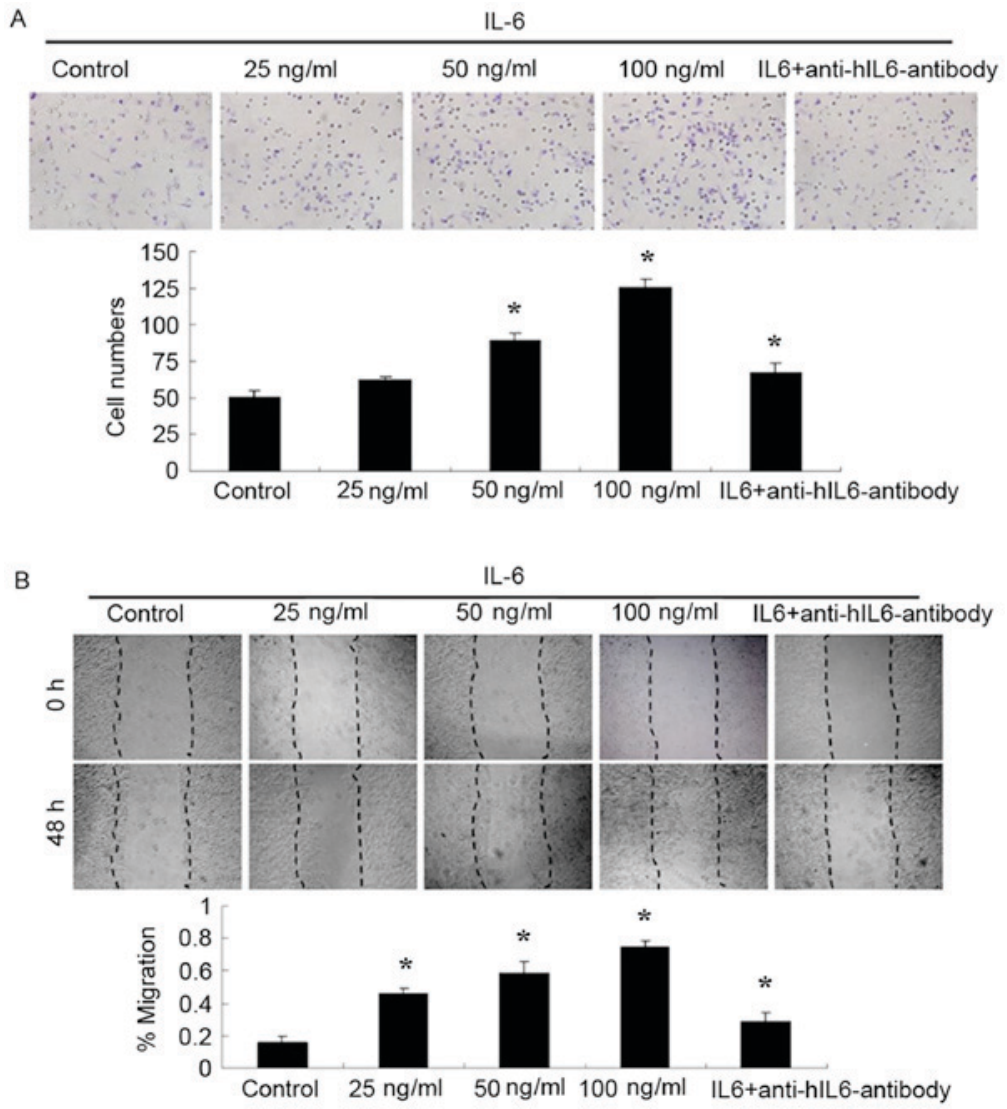

Figure 5. Migration of ACP cells treated with IL-6 $(0-100 \mathrm{ng} / \mathrm{ml})$ or IL-6 $(100 \mathrm{ng} / \mathrm{ml}) /$ anti-hIL-6-antibody $(10 \mu \mathrm{g} / \mathrm{ml})$. Migration if ACP cell cultures was enhanced by IL-6 treatment in a dose-dependent manner in (A) Boyden chamber (magnification, x400) and (B) wound healing assays (magnification, x200), however, the IL-6-blocking monoclonal antibody significantly decreased the migration of ACP cell cultures. Data are presented as the mean \pm standard deviation. "P<0.05 vs. control. ACP, adamantinomatous craniopharyngiomas; IL-6, interleukin-6; h, human. 


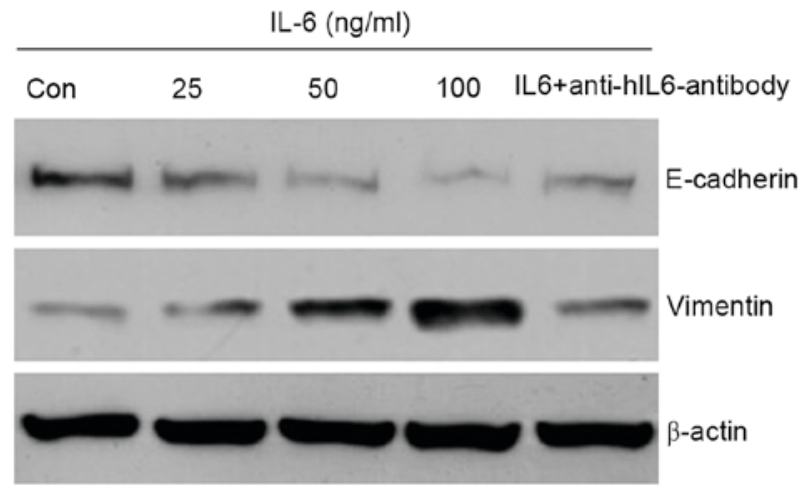

Figure 6. Western blot analysis was performed to detect the protein expression of vimentin and E-cadherin in ACP cells, which were treated with various levels of IL-6 $(0-100 \mathrm{ng} / \mathrm{ml})$ and IL-6 $(100 \mathrm{ng} / \mathrm{ml}) /$ anti-hIL-6-antibody $(10 \mu \mathrm{g} / \mathrm{ml})$ in serum-free culture medium. Vimentin was upregulated and simultaneously associated with the downregulation of E-cadherin in an IL-6 dose-dependent manner. However, the IL-6-blocking monoclonal antibody significantly reversed these protein expression patterns. ACP, adamantinomatous craniopharyngiomas; IL-6, interleukin-6; h, human.

whereas E-cadherin expression was downregulated in an IL-6 dose-dependent manner.

\section{Discussion}

CPs are benign epithelial tumors of the sellar region; their aggressive behavior and potential for adhesion to adjacent brain structures are conducive to significant regrowth or recurrence even following total tumor removal. Histologically, intense fibrillary gliosis rich in Rosenthal fibers and infiltration of inflammatory cells are frequently present in the surrounding parenchyma in CPs, particularly in ACP (13).

Chronic inflammation, which is implicated in all stages of carcinogenesis, including initiation, promotion and progression, represents a major pathological basis for the majority of human malignancies. In the present study, CP inflammation was associated with pathological classification, the rate of total resection, calcification and postoperative HSS. Within the tumor microenvironment, a number of proinflammatory mediators, such as cytokines, participate in complex inflammatory signaling pathways that facilitate the extravasation of tumor cells through the stroma, thereby stimulating tumor progression (14). Results from the present study, as well as a study by Mori et al (4), strongly implicated IL-6 as the key molecule for ACP inflammation.

IL-6 is a multifunctional cytokine that exerts pleiotropic activities on various cell types. It is usually produced at local tissue sites during infection, trauma or in tumors. IL- 6 can bind to IL-6R (classic-signaling) and/or sIL-6R (trans-signaling) to form a complex that activates aberrant signaling pathways, which contribute to the promotion of tumorigenesis and tumor angiogenesis, migration and invasion (15). Numerous biological activities are mediated by the IL- 6 cytokine. Mori et al (4) proposed that IL-6 may serve an important role in the inflammatory reaction that occurs in the interface between the $\mathrm{CP}$ and brain parenchyma. However, the biological activities and mechanisms involved in $\mathrm{CP}$ cells remain unclear.

The present study demonstrated that the IL-6, IL-6R and GP130 proteins are present in some ACP tissues. In addition,
sIL-6R was observed to accumulate in the supernatant of ACP cells, ACP fibroblasts and ACP cystic fluid. Therefore, autocrine and/or paracrine sources of IL-6 may serve an important role in regulation of the biological characteristics of ACP. IL-6 did not affect the viability of ACP cells, however, IL-6 promoted the migration of ACP cells following a primary ACP culture assay. Depletion of IL- 6 from the ACP medium abolished the IL- 6 mediated stimulatory effect on ACP cell migration.

In addition, the present study revealed that EMT may serve a role in the pathogenesis and development of CPs, particularly ACP. Overexpression of vimentin and decreased expression of E-cadherin are closely associated with tumor recurrence and poor postoperative hypothalamic function in patients with CP. It has been well documented that an IL-6-mediated EMT phenotype in some cancer cells, such as breast cancer and biliary tract cancer cells, is clinically associated with unfavorable outcomes in numerous human carcinoma types (6). In the present study, a rapid dose-dependent increase in vimentin and decrease in E-cadherin was observed following ACP cell stimulation with IL-6, and the expression of these two proteins was reversed following IL-6 blocking with a neutralizing antibody. Therefore, EMT may be involved in IL-6-promoted migration.

The IL-6 signaling network has been targeted with several therapeutic antagonists in numerous human cancer preclinical and clinical trials; however, this strategy has yet to be applied for CP treatments. The present study observed an IL-6-mediated EMT phenotype in ACP cells, which is clinically associated with unfavorable outcomes in several human carcinoma types. Further studies are required to fully elucidate the mechanism of action of IL-6 and the specific signal transduction pathways involved in ACP progression. However, the results may support further evaluation of anti-IL-6 therapies in CPs, particularly ACP.

In conclusion, a local inflammatory state between tumor cells and parenchyma is generated by the enhanced infiltration of leukocytes and tumor cell-derived cytokines, particularly IL-6, at the adjacent tissue in CPs. To the best of our knowledge, the present study is the first to demonstrate that IL-6 may promote migration in vitro via classic- and trans-signaling pathways by inducing EMT in ACP. Therefore, anti-IL-6 strategies targeting its downstream pathways and proteins may be promising for the treatment of ACP.

\section{Acknowledgements}

The present study was funded by the Natural Science Foundation of Guangdong province (grant no. S2012020010939) and the Natural Science Foundation of Luzhou Medical College (grant no. 2013ZRZD005).

\section{References}

1. Karavitaki N, Cudlip S, Adams CB and Wass JA: Craniopharyngiomas. Endocr Rev 27: 371-397, 2006.

2. Hanahan D and Weinberg RA: Hallmarks of cancer: The next generation. Cell 144: 646-674, 2011.

3. Satoh H, Uozumi T, Arita K, Kurisu K, Hotta T, Kiya K, Ikawa F, Goishi J and Sogabe T: Spontaneous rupture of craniopharyngioma cysts: A report of five cases and review of the literature. Surg Neurol 40: 414-419, 1993. 
4. Mori M, Takeshima $\mathrm{H}$ and Kuratsu J: Expression of interleukin-6 in human craniopharyngiomas: A possible inducer of tumor-associated inflammation. Int J Mol Med 14: 505-509, 2004.

5. Walter M, Liang S, Ghosh S, Hornsby PJ and Li R: Interleukin 6 secreted from adipose stromal cells promotes migration and invasion of breast cancer cells. Oncogene 28: 2745-2755, 2009.

6. Sullivan NJ, Sasser AK, Axel AE, Vesuna F, Raman V, Ramirez N, Oberyszyn TM and Hall BM: Interleukin-6 induces an epithelial-mesenchymal transition phenotype in human breast cancer cells. Oncogene 28: 2940-2947, 2009.

7. Qi ST, Zhou J, Pan J, Zhang C, Silky C and Yan XR Epithelial-mesenchymal transition and clinicopathological correlation in craniopharyngioma. Histopathology 61: 711-725, 2012.

8. Qi S, Huang G, Pan J, Li J, Zhang X, Fang L, Liu B, Meng W, Zhang Y and Liu X: Involvement of osteopontin as a core protein in craniopharyngioma calcification formation. J Neurooncol 98: 21-30, 2010.

9. Fahlbusch R, Honegger J, Paulus W, Huk W and Buchfelder M: Surgical treatment of craniopharyngiomas: Experience with 168 patients. J Neurooncol 90: 237-250, 1999.
10. Hölsken A, Buchfelder M, Fahlbusch R, Blümcke I and Buslei R: Tumour cell migration in adamantinomatous craniopharyngiomas is promoted by activated Wnt-signalling. Acta Neuropathol 119: 631-639, 2010.

11. Louis DN, Ohgaki H, Wiestler OD, Cavenee WK, Burger PC, Jouvet A, Scheithauer BW and Kleihues P: The 2007 WHO classification of tumours of the central nervous system. Acta Neuropathol 11: 97-109, 2007.

12. Smith PC, Hobisch A, Lin DL, Culig Z and Keller ET: Interleukin- 6 and prostate cancer progression. Cytokine Growth Factor Rev 12: 33-40, 2001.

13. Karavitaki N and Wass JA: Craniopharyngiomas. Endocrinol Metab Clin North Am 37: 173-193, ix-x, 2008.

14. Balkwill $\mathrm{F}$ and Mantovani A: Inflammation and cancer: Back to Virchow? Lancet 357: 539-545, 2001.

15. Rose-John S, Scheller J, Elson G and Jones SA: Interleukin-6 biology is coordinated by membrane-bound and soluble receptors: Role in inflammation and cancer. J Leukoc Biol 80: 227-236, 2006. 